\title{
Different contribution of class II HLA in fulminant and typical autoimmune type 1 diabetes mellitus
}

\author{
A. Imagawa - T. Hanafusa • Y. Uchigata • \\ A. Kanatsuka • E. Kawasaki • T. Kobayashi • \\ A. Shimada • I. Shimizu • T. Maruyama - H. Makino
}

Published online: 9 January 2008

(C) Springer-Verlag 2007

\section{Erratum to: Diabetologia (2005) 48:294-300 \\ DOI 10.1007/s00125-004-1626-x}

Unfortunately there were miscalculations in Tables 3 and 4 of this paper. The corrected tables are reproduced below (with the amended values in colour), along with the corrected paragraph from the Results. The authors believe that these miscalculations are minimal and do not change the conclusion of the paper.

The online version of the original article can be found at http://dx.doi. org/10.1007/s00125-004-1626-x.

\section{A. Imagawa}

Department of Internal Medicine and Molecular Science,

Graduate School of Medicine, Osaka University,

Osaka, Japan

A. Imagawa $\cdot$ T. Hanafusa $(\bowtie)$

First Department of Internal Medicine, Osaka Medical College,

2-7 Daigaku-machi,

569-8686 Takatsuki, Japan

e-mail: hanafusa@poh.osaka-med.ac.jp

Y. Uchigata

Diabetes Center,

Tokyo Women's Medical University School of Medicine,

Tokyo, Japan

\section{A. Kanatsuka}

Diabetes Center, Kasori Hospital,

Chiba, Japan

\section{E. Kawasaki}

Department of Metabolism/Diabetes and Clinical Nutrition, Nagasaki University Hospital of Medicine and Dentistry,

Nagasaki, Japan

\section{Serological typing of HLA-DR}

The allele frequency of HLA-DR4 was $44.7 \%$ in fulminant diabetic patients, $29.1 \%$ in autoimmune diabetic patients, and $21.8 \%$ in healthy control subjects. HLA-DR4 occurred at a significantly greater frequency in fulminant diabetic patients than in healthy controls $(p=2.85 \mathrm{E}-09, p c=2.57 \mathrm{E}-08$, [OR 2.90]), but there was no significant difference in the frequency of HLA-DR4 between autoimmune type 1 diabetic patients and healthy controls. The HLA-DR2 allele was observed in only $1.0 \%$ of autoimmune type 1 diabetes, but in $10.1 \%$ of fulminant diabetic patients, with

\section{T. Kobayashi}

Third Department of Internal Medicine,

University of Yamanashi School of Medicine,

Tamaho, Japan

A. Shimada

Department of Internal Medicine,

Keio University School of Medicine,

Tokyo, Japan

I. Shimizu

Internal Medicine, Ehime Prefectural Central Hospital,

Ehime, Japan

T. Maruyama

Department of Internal Medicine,

Saitama Social Insurance Hospital,

Saitama, Japan

H. Makino

Department of Laboratory Medicine,

Ehime University School of Medicine,

Ehime, Japan 
Table 3 HLA-DR-DQ haplotype frequency in type 1 diabetic and normal control subjects

\begin{tabular}{|c|c|c|c|c|c|c|c|c|c|c|c|}
\hline & \multirow{2}{*}{$\begin{array}{l}\text { Fulminant } \\
(n=182)\end{array}$} & \multirow{2}{*}{$\begin{array}{c}\text { Autoimmune } \\
\quad(n=162)\end{array}$} & \multirow{2}{*}{$\begin{array}{l}\text { Control } \\
(n=380)\end{array}$} & \multicolumn{3}{|c|}{ Fulminant vs control } & \multicolumn{3}{|c|}{ Autoimmune vs control } & \multicolumn{2}{|c|}{ Fulminant vs autoimmune } \\
\hline & & & & $p$ & $p c$ & OR & $p$ & $p c$ & OR & $p$ & $p c$ \\
\hline $1-1$ & $2.7(5)$ & $5.6(9)$ & $7.1(27)$ & - & - & - & - & - & - & - & - \\
\hline $2-1$ & $8.2(15)$ & $1.2(2)$ & $18.7(71)$ & 0.001 & 0.014 & 0.39 & $6.56 \mathrm{E}-10$ & 8.53E-09 & 0.054 & 0.0024 & 0.031 \\
\hline $3-2$ & $0.5(1)$ & $1.2(2)$ & $0.3(1)$ & - & - & - & - & - & - & - & - \\
\hline $4-1$ & $0.0(0)$ & $0.6(1)$ & $1.6(6)$ & - & - & - & - & - & - & - & - \\
\hline $4-3$ & $4.4(8)$ & $4.3(7)$ & $8.2(31)$ & - & - & - & - & - & - & - & - \\
\hline $4-4$ & $41.8(76)$ & $22.8(37)$ & $12.1(46)$ & $1.47 \mathrm{E}-15$ & $1.91 \mathrm{E}-14$ & 5.21 & 0.0015 & 0.019 & 2.15 & 0.00019 & 0.0025 \\
\hline $5-3$ & $2.7(5)$ & $4.3(7)$ & $6.3(24)$ & - & - & - & - & - & - & - & - \\
\hline $6-1$ & $12.6(23)$ & $11.7(19)$ & $9.2(35)$ & - & - & - & - & - & - & - & - \\
\hline $6-3$ & $1.6(3)$ & $0.0(0)$ & $3.2(12)$ & - & - & - & - & - & - & - & - \\
\hline $8-1$ & $1.6(3)$ & $1.9(3)$ & $9.2(35)$ & 0.00047 & 0.0061 & 0.17 & 0.0014 & 0.019 & 0.19 & - & - \\
\hline $8-3$ & $1.1(2)$ & $3.7(6)$ & $3.7(14)$ & - & - & - & - & - & - & - & - \\
\hline $8-4$ & $1.1(2)$ & $0.6(1)$ & $4.0(15)$ & - & - & - & - & - & - & - & - \\
\hline $9-3$ & $19.8(36)$ & $39.5(64)$ & $15.0(57)$ & - & - & - & $3.57 \mathrm{E}-10$ & 4.64E-09 & 3.70 & - & - \\
\hline Others & $1.6(3)$ & $2.5(4)$ & $1.6(6)$ & & & & & & & & \\
\hline
\end{tabular}

Data are $\%(n)$. Others contain a rare haplotype, whose total frequencies in all of patients and controls were less than three

a significant difference in the frequency of HLA-DR2 between these two groups $(p=0.0000039, p c=0.000035)$. HLA-DR2 was less frequent in fulminant diabetes and autoimmune diabetes than in healthy controls $(p=0.0027$, $p c=0.024,[\mathrm{OR} 0.47]$ and $p=2.61 \mathrm{E}-12, p c=2.35 \mathrm{E}-11$,
[OR 0.04], respectively). HLA-DR9 was significantly more frequent in autoimmune type 1 diabetes than in healthy controls ( $p=5.39 \mathrm{E}-12, p c=4.85 \mathrm{E}-11$, [OR 3.91]), but was not different in fulminant type 1 diabetes compared with controls. The HLA-DR8 allele was less

Table 4 Combination of HLA-DR-DQ haplotype in type 1 diabetic and control subjects

\begin{tabular}{|c|c|c|c|c|c|c|c|c|c|c|c|}
\hline & \multirow{2}{*}{$\begin{array}{l}\text { Fulminant } \\
\qquad(n=91)\end{array}$} & \multirow{2}{*}{$\begin{array}{l}\text { Autoimmune } \\
\qquad(n=81)\end{array}$} & \multirow{2}{*}{$\begin{array}{l}\text { Control } \\
(n=190)\end{array}$} & \multicolumn{3}{|c|}{ Fulminant vs control } & \multicolumn{3}{|c|}{ Autoimmune vs control } & \multicolumn{2}{|c|}{ Fulminant vs autoimmune } \\
\hline & & & & $p$ & $p c$ & OR & $p$ & $p c$ & OR & $p$ & $p c$ \\
\hline $4-4 / 4-4$ & $17.6(16)$ & $6.2(5)$ & $1.6(3)$ & 5.74E-07 & $2.87 \mathrm{E}-06$ & 13.3 & - & - & - & 0.034 & NS \\
\hline $4-4 / X$ & $33.0(30)$ & $21.0(17)$ & $14.7(28)$ & 0.00041 & 0.002 & 2.85 & - & - & - & - & - \\
\hline $4-4 / 9-3$ & $15.4(14)$ & $12.3(10)$ & $6.3(12)$ & 0.014 & NS & 2.70 & - & - & - & - & - \\
\hline $9-3 / 9-3$ & $3.3(3)$ & $22.2(18)$ & $2.1(4)$ & - & - & - & $2.08 \mathrm{E}-07$ & $1.04 \mathrm{E}-06$ & 13.3 & 0.00014 & 0.0007 \\
\hline $9-3 / X$ & $15.4(14)$ & $22.2(18)$ & $19.5(37)$ & - & - & - & - & - & - & - & - \\
\hline$X / X$ & $15.4(14)$ & $16.0(13)$ & $55.8(106)$ & & & & & & & & \\
\hline
\end{tabular}

Data are $\%(n)$

NS not significant, $X$ neither DR4-DQ4 nor DR9-DQ3 
frequent in fulminant and autoimmune type 1 diabetes than in healthy controls $(p=0.000059, p c=0.00052$, [OR 0.25 ] and $p=0.0024, p c=0.021$, [OR 0.41], respectively). HLA-DR1 and -DR5 were also less frequent in fulminant type 1 diabetes than in healthy controls $(p=0.017, p c>0.05$, [OR 0.34$]$ and $p=0.0082, p c>0.05$, [OR 0.29], respec- tively), but there were no significant differences between fulminant and autoimmune type 1 diabetes or between autoimmune diabetes and healthy controls. The differences between phenotypic and allele frequencies in HLA-DR3, -DR6, and -DR7 among the three groups were not significant. 